\title{
Nuclear M-CSF Accelerates DNA Replication and Cell Proliferation in HeLa Human Cervical Cancer Cells
}

\author{
Jian Tu ${ }^{1}$, Ting Xiong ${ }^{1}$, Shujing Teng ${ }^{1}$, Zhigang Zhou $^{2}$, Xiaoyong Lei ${ }^{1}$, Shengsong Tang ${ }^{1}{ }^{*}$ \\ ${ }^{1}$ Department of Pharmacology, University of South China, Hengyang, China \\ ${ }^{2}$ Department of Anesthesiology, University of South China, Hengyang, China
}

Email address:

tangss111@aliyun.com (Shengsong Tang)

${ }^{*}$ Corresponding author

\section{To cite this article:}

Jian Tu, Ting Xiong, Shujing Teng, Zhigang Zhou, Xiaoyong Lei, Shengsong Tang. Nuclear M-CSF Accelerates DNA Replication and Cell Proliferation in HeLa Human Cervical Cancer Cells. Cell Biology. Vol. 5, No. 1, 2017, pp. 1-7. doi: 10.11648/j.cb.20170501.11

Received: January 31, 2017; Accepted: February 20, 2017; Published: March 4, 2017

\begin{abstract}
Macrophage colony-stimulating factor (M-CSF), also named colony-stimulating factor-1 (CSF-1), plays an important role in the process of proliferation and differentiation of the monocyte/macrophage lineage cells. Commonly, it is not easy to measure the expression of cellular M-CSF. However, recent studies have shown that M-CSF can be expressed at a high level in the cytoplasm and nuclei of some kinds of malignant tumors, which related to the poor prognosis. To explore the role and mechanism of nuclear M-CSF, in the present study we constructed the $\mathrm{pCMV} / \mathrm{nuc} / \mathrm{M}-\mathrm{CSF}$ vector and transfected it into human HeLa nuclei. The results from our previous study indicated, M-CSF was stably expressed in HeLa nuclei, which were used as a model to determine the nuclear effects of M-CSF. There was a higher percentage of replicating nuclei in the transfected $\mathrm{pCMV} / \mathrm{nuc} / \mathrm{M}$-CSF HeLa cells both in phase G1 and S. According to the data from the cell doubling time, antisense oligonucleotides and the experiments of the transplanted tumor in nude mice, nuclear M-CSF could promote the cell proliferation of HeLa cells both in vivo and in vitro. In conclusion, nuclear M-CSF could accelerate DNA replication and cell proliferation of cervical carcinoma.
\end{abstract}

Keywords: Microphage Colony-Stimulating Factor (M-CSF), HeLa Nuclei, DNA Replication, Cell Proliferation

\section{Introduction}

Cervical cancer is one of the most frequent diseases of the reproductive organs and its morbidity rate is constantly increasing. The development of cervical cancer seems to be highly associated with human papillomavirus (HPV) infection [1]. Although HPV is the major etiological agent of cervical cancer, yet the viral infection alone is not sufficient for cancer progression [2]. Lawicki et al. shows that different tumor markers may also be useful in the diagnosis of cervical cancer [3], including squamous cell carcinoma antigen (SCC-Ag), tissue polypeptide antigen (TPA), as well as some cytokines such as vascular endothelial growth factor (VEGF), granulocyte colony-stimulating factor (G-CSF) and macrophage colony-stimulating factor (M-CSF), and so on. It has been shown that HPV enhanced expression of IL-1 $\beta$ and IL-8, while G-CSF and M-CSF did not change. M-CSF, also called colony-stimulating factor- 1 (CSF-1), is important in controlling the survival, proliferation and differentiation of the monocyte/macrophage lineage cells both in vivo and in vitro [4]. As an extracellular signaling molecule, M-CSF swims in body fluids in normal physiological conditions. It is binding with the extracellular matrix or exists on the surface of cell membrane only during the embryonic period. When the function process is completed, M-CSF will be endocytosised and degradated in the cells. Therefore, it is not easy to measure the expression of cellular M-CSF $[5,6]$. Encoded by a single gene, M-CSF contains five mature alternative transcripts. Under pathological conditions, there are at least three isoforms of M-CSF [7]: soluble M-CSF (s-M-CSF), membrane-bound M-CSF (m-M-CSF) and proteglycan-bound M-CSF (PG-M-CSF), which have been generated from these transcripts through co- and post-translational modifications. Different kinds of M-CSF isoforms are found in different cell types and under various conditions of stimulation.

Recently, the potential role and mechanism of cellular 
M-CSF have been focused in leukemia, cervical cancer, endometrial carcinomas and breast cancer, and so on [8-11]. The data of Nameer Kirma showed the induction of cellular $\mathrm{M}-\mathrm{CSF}$ and its receptor (c-fms) in cervical carcinomas and suggest that blocking CSF-1/c-fms might be a viable therapeutic strategy in the treatment of cervical cancer [12]. Moreover, intracellular M-CSF (including cytosol or nuclear M-CSF) was also identified pathologically in the cell lines of leukemia, breast cancer and Hepatocellular Carcinoma (HCC)[13-15]. Interestingly, nuclear M-CSF (nM-CSF) was found only in some tumor cells other than normal cells. The result of pathological analysis on nM-CSF showed that, the appearance of nM-CSF suggested poor prognosis and increased metastasis tendency in some carcinomas [16, 17]. But the function and mechanism of nM-CSF have not been elucidated. To further explore the role and mechanism of nM-CSF, we have constructed $\mathrm{pCMV} /$ nuc/M-CSF vector and transfected it into human HeLa nuclei [18].

\section{Method}

\subsection{Materials}

E. coli $\mathrm{DH} 5 \alpha$ was kept in our laboratory, $\mathrm{pCMV} / \mathrm{myc} / \mathrm{nuc}$ was purchased from Invitrogen (Carlsbad, CA), and pET32c+/M-CSF was kindly provided by Doctor Cao ZY. Plasmid pCMV/nuc/M-CSF was described previously [19]. HeLa cells were purchased from Zhongshan Medical College cell bank (Guangzhou, China).

\subsection{Cell Culture and Transfection}

A total of $1 \times 10^{5} \mathrm{HeLa}$ cells were seeded into the 24 -well tissue culture plate. The cell monolayer was washed with pre-warmed sterile phosphate-buffered saline (PBS) the next day (when the cells were $50 \%-80 \%$ confluent). The cells were transfected by using Lipofectamine ${ }^{\mathrm{TM}} 2000$ (Invitrogen, Carlsbad, CA) according to the manufacturer's protocol. The cells were placed in the selection medium containing different concentration $(200,400,600,800$, or $1000 \mu \mathrm{g} / \mathrm{mL})$ of $\mathrm{G} 418$ (Invitrogen, Carlsbad, CA). Fourteen days later, the cells were harvested for analysis.

\subsection{DNA Replication Assessment in Vivo Using Bromo-Deoxyuridine (BrdU)}

Transfected and controlled HeLa cells $\left(2-5 \times 10^{5}\right.$ cells $)$ were seeded into the 6 -well tissue culture plate (with a $24 \mathrm{~mm} \times 24$ $\mathrm{mm}$ coverslip inside). After $48 \mathrm{~h}$, the medium was replaced with fresh medium containing $50 \mu \mathrm{M}$ BrdU, and the cells were cultured at $37^{\circ} \mathrm{C}$ for $30 \mathrm{~min}$. Then, the cells were washed with ice-cold PBS, fixed with $4 \%$ formaldehyde at room temperature for $10 \mathrm{~min}$, washed with PBS twice, and permeabilized with $0.5 \%$ TritonX-100 in PBS on ice for 5-10 min. After being washed with PBS and rinsed with sterilized distilled water, the cells (on the coverslip) were lyzed by incubation with $2 \mathrm{~mol} / \mathrm{L} \mathrm{HCl}$ at $37^{\circ} \mathrm{C}$ for $1 \mathrm{~h}$ and then neutralized with $0.1 \mathrm{~mol} / \mathrm{L} \mathrm{Na}_{2} \mathrm{~B}_{4} \mathrm{O}_{7}$. The coverslips were then incubated with anti-BrdU $\mathrm{mAb}$ (diluted at 1:200) in
$0.5 \% \mathrm{BSA} / 0.1 \%$ Tween $20 / \mathrm{PBS}$ overnight. The coverslips were washed with the BSA/Tween/PBS solution, and incubated with FITC-conjugated secondary antibody (diluted at 1:50) for $2 \mathrm{~h}$ at room temperature. In a dark room, the coverslips were washed with PBS, incubated with $20 \mu \mathrm{g} / \mathrm{mL}$ propidium iodide at room temperature for $3 \mathrm{~min}$ and then examined by fluorescence microscopy. Replication in vivo was analyzed by using BrdU. After the absorbing of BrdU, BrdUTP was produced as phosphorylation. The precursor BrdUTP could be incorporated to DNA instead of dTTP. Then, with anti-BrdU monoclonal primary antibody and FITC marked secondary antibody tested, nuclei in duplicate present green fluorescent. After Propidium Iodide (PI) redyeing, the nuclei were red.

\subsection{Mammalian Cell-Free DNA Replication System in Vitro}

\subsubsection{Cell Synchronization}

The cells were synchronized in block by using a culture medium containing $2.5 \mathrm{mM}$ thymidine (Sigma) according to the methods previously described [20]. The process was verified by flow cytometry.

\subsubsection{Preparation of Nuclei and Cell-Free Extracts}

The cells were washed twice with ice-cold hypotonic buffer (20 mM potassium-HEPES [pH 7.8], $5 \mathrm{mM}$ potassium acetate, $0.5 \mathrm{mM} \mathrm{MgCl}_{2}$, and $0.5 \mathrm{mM}$ DTT). All subsequent steps were carried out at $4^{\circ} \mathrm{C}$. The cells were allowed to swell for $10 \mathrm{~min}$ in $20 \mathrm{~mL}$ hypotonic buffer per plate and the excess buffer was removed. At this stage, mitotic cells were lost because they detached from the substratum under hypotonic conditions. The interphase cells still attaching to the substratum that were scraped off the plates and disrupted with 25 strokes in a dounce homogenizer (Wheaton) using a loose-fitting pestle. The nuclei were pelleted at $1,700 \mathrm{~g}$ for $5 \mathrm{~min}$. The supernatant was used to prepare the cytosolic extract. Pelleted nuclei were resuspended and washed three times in PBS and finally pelleted at $1,700 \mathrm{~g}$ for $5 \mathrm{~min}$. The excess supernatant was removed, and the pelleted nuclei were resuspended in the residual volume. The concentration of nuclei were determined with a hemocytometer.

\subsubsection{DNA Synthesis in Vitro}

Reactions were carried out in $50 \mu \mathrm{L}$ of a buffered nucleotide mix (yielding final concentrations of $40 \mathrm{mM} \mathrm{K-HEPES} \mathrm{[pH}$ 7.8]; $7 \mathrm{mM} \mathrm{MgCl} 2 ; 3 \mathrm{mM}$ ATP; $0.1 \mathrm{mM}$ of each of GTP, CTP, UTP; 3 mM ATP; $0.25 \mu \mathrm{M}$ biotin-16-dUTP; 0.5 mM DTT; 40 $\mathrm{mM}$ creatine phosphate; and $5 \mu \mathrm{g}$ phosphocreatine kinase). Nuclei $\left(1 \times 10^{5}\right.$ per reaction) were mixed with the buffered nucleotide mix on ice and the reaction was started by transferring the tube to $37^{\circ} \mathrm{C}$ for $2 \mathrm{~h}$. After centrifugation at $100 \mathrm{~g}$ for $5 \mathrm{~min}$ and washed with PBS twice, the nuclei were transferred onto the coverslips precoated with Poly-L-Lysine. The coverslips were fixed with $4 \%$ formaldehyde at room temperature for $10 \mathrm{~min}$, washed with PBS twice, and then permeabilized with $0.5 \%$ TritonX-100/PBS on ice for 5 to 10 min. The coverslips were washed with PBS twice and incubated with FITC-conjugated anti-avidin (diluted at 1: 64 with $1 \% \mathrm{BSA} / \mathrm{PBS}$ ) for $2 \mathrm{~h}$ at room temperature. In the dark 
room, the coverslips were washed with PBS and examined by fluorescence microscopy.

\subsection{Cell Kinetic Analysis}

\subsubsection{Counting the Cell Doubling Time}

The cells were harvested and counted continuously from the 1 st to 7 th day to take averaging by three wells each time and three times each well. The calculation formula of the doubling time was as follows: Doubling time (d) $=[$ TIME (end) - TIME (begin)]/ $\log _{2}$ [Cell concentration (end)/Cell concentration (begin)].

\subsubsection{Cell Proliferation Assay}

The cell proliferative rate was determined by Methylthiazolyldiphenyl-tetrazolium bromide (MTT) assay. The three kinds of HeLa cells $\left(1 \times 10^{4}\right.$ per well) were cultured in 96-well plates. Thereafter, $20 \mu \mathrm{L}$ MTT solution was added to each well. After continued incubation for $4 \mathrm{~h}$, the supernatant was discarded and $200 \mu \mathrm{L}$ DMSO was added. Once the blue crystals were dissolved, the optical density (OD) was measured at $490 \mathrm{~nm}$ using a plate microreader (Tecan Spectra, Wetzlar, Germany). The experiments were performed in triplicate. The proliferation rate was determined using the following formula: Cell proliferation $(\%)=\mathrm{OD}$ of the experimental samples/OD of the control $\times 100 \%(n=3$, mean \pm SD).

\subsubsection{M-CSF-specific Antisense Oligonucleotide}

M-CSF-specific antisense oligonucleotide (nucleotide sulfur generation modificated) was 5'-gcgccecgcgeggtcat-3' and random fragment contrast (nucleotide sulfur generation modificated) sequence was 5'-cgcacgcctgctgctcg-3', synthesed by the Shanghai biological engineering co., LTD. The cell concentration was adjusted for $5 \times 10^{4} \mathrm{cells} / \mathrm{mL}$ to vaccinate 96-well plates, each well $180 \mu \mathrm{L}$. With RPMI1640 medium dissoluted, (1, 3, 5, 7, $9 \mathrm{nmol} / \mathrm{L})$ antisense oligonucleotides were joined every well for $44 \mathrm{~h}$, and $20 \mu \mathrm{L}$ MTT $(5 \mathrm{mg} / \mathrm{mL})$ was added to cultivate for $4 \mathrm{~h}$, then $200 \mathrm{uL}$ DMSO was added. The inhibitory rate of M-CSF-specific antisense oligonucleotide was determined using the following formula: Inhibitory rate $(\%)=$ $\left(\mathrm{OD}_{\mathrm{CONTROL}}-\mathrm{OD}_{\mathrm{ASODN}}\right) / \mathrm{OD}_{\mathrm{CONTROL}} \times 100 \%$.

\subsubsection{Xenograft Experiments}

A suspension of $2 \times 10^{6} \mathrm{HeLa}$ cells in a total volume was injected subcutaneously into the right dorsal flank of the nude mice. Following the engraftment, the mice were assigned into three groups with three kinds of HeLa cells mentioned above treated respectively. The animals were maintained under sterile conditions in individually vented cages. The mice were raised for one month. At the end, the animals were sacrificed and tumors were excised.

\subsection{Statistics}

Statistics were calculated with the SPSS 12.0 software package. The chi-square test was applied for enumeration data. Analysis of variance (ANOVA) was applied for comparison of the means of two or multiple groups of measurements, and the Student-Newman-Keuls (SNK) test was used for further group comparison. For all of the analyses, a $P$-value $<0.05$ was considered statistically significant.

\section{Results}

\subsection{Analysis of Replication in Vivo}

The nuclei in duplicate present green fluorescent as Figure $1 \mathrm{~A}$ (a) showed; after Propidium Iodide (PI) redyeing, the nuclei were red ( $b$ in Figure 1). The total of 300 to 400 nuclei were counted to determine the percentage of replicating nuclei. The results were that the percentage of replicating nuclei was $40.13 \pm 1.02 \%$ in HeLa-M cells, which was much higher than $20.54 \pm 0.52 \%$ in HeLa cells or $21.23 \pm 0.63 \%$ in HeLa-C cells. As a result, the DNA replicating ability of HeLa-M was stronger than the other two groups ( $\mathrm{n}=3, * P<0.05$ vs the other two groups). There was a higher percentage of replicating nuclei in the transfected pCMV/nuc/M-CSF HeLa cells (HeLa-M) compared with the other two groups $(\mathrm{n}=3, P<0.05$, Figure $1 \mathrm{~B})$.

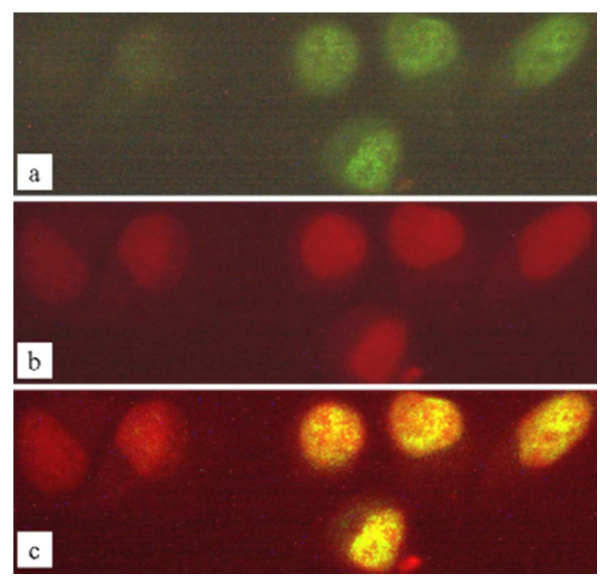

A

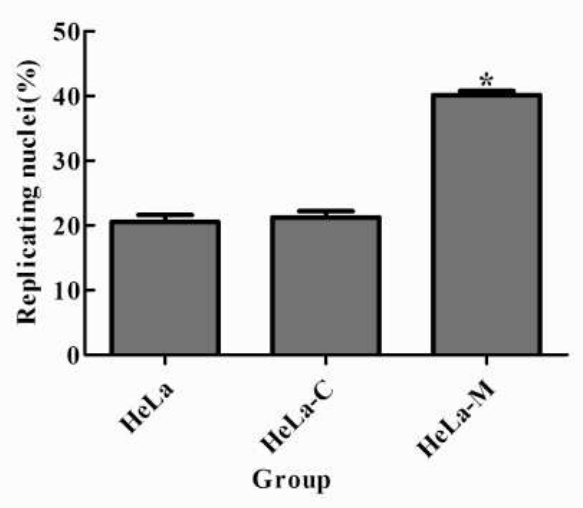

B

Figure 1. The intracellular DNA replicating ability of the three groups.

A) The intracellular DNA replication by BrdU labeling. a: Replicating nuclei; b: Nuclei (PI after stained); c: The merged of the two ahead $(\times 400)$.

B) The percentage of replicating nuclei in the three groups. ${ }^{*} P<0.05$ vs the other two groups $(n=3)$;

HeLa: untransfected HeLa cells; HeLa-C: transfected pCMV/myc/nuc HeLa cells; HeLa-M: transfected pCMV/nuc/M-CSF HeLa cells. 


\subsection{Analysis of Replication in Vitro Using a Cell-Free DNA System}

To identify whether M-CSF could affect the initiation or elongation stage of DNA replication or not, a cell-free DNA replication system was used in vitro. The cells were synchronized at stage G1/S using a thymidine double-block and verified by flow cytometry. Then, the cells in stage G1 and $\mathrm{S}$ were obtained (Table 1).

Table 1. Statistical results of cell synchronization blocked doubly by Thymidine for different time. ( $\overline{\boldsymbol{x}} \pm S, \%)$.

\begin{tabular}{lllllll}
\hline cell cycle & $\mathbf{0 h}$ & $\mathbf{1 h}$ & $\mathbf{3 h}$ & $\mathbf{5 h}$ & $\mathbf{7 h}$ & $\mathbf{9 h}$ \\
\hline G1 & $95.60 \pm 1.27$ & $96.25 \pm 1.48^{*}$ & $94.35 \pm 1.91$ & $1.30 \pm 0.71$ & $1.25 \pm 0.07$ & $0.09 \pm 0.85$ \\
G2/M & $1.50 \pm 0.57$ & $1.90 \pm 1.13$ & $0.45 \pm 0.36$ & $0.40 \pm 0.14$ & $0.00 \pm 0.00$ & $62.00 \pm 8.48$ \\
S & $2.95 \pm 0.64$ & $1.85 \pm 2.62$ & $5.20 \pm 2.27$ & $98.35 \pm 0.49$ & $98.75 \pm 0.07 \#$ & $37.10 \pm 9.33$ \\
\hline
\end{tabular}

*: Release of the thymidine double-block for $1 \mathrm{~h}$ yielded $96.25 \%$ of the cells in stage G1;

\#: Release of the thymidine double-block for $7 \mathrm{~h}$ yielded $98.75 \%$ of the cells in stage $\mathrm{S}$.

The total of 300 to 400 nuclei were counted to determine the percentage of replicating nuclei. The results showed that both in phase $\mathrm{G} 1$ and $\mathrm{S}$, the percentage of replicating nuclei in the HeLa-M group was higher than that in the other two groups ( $\mathrm{n}=3, P<0.05$, Figure $2 \mathrm{~A}$ and $\mathrm{B})$.

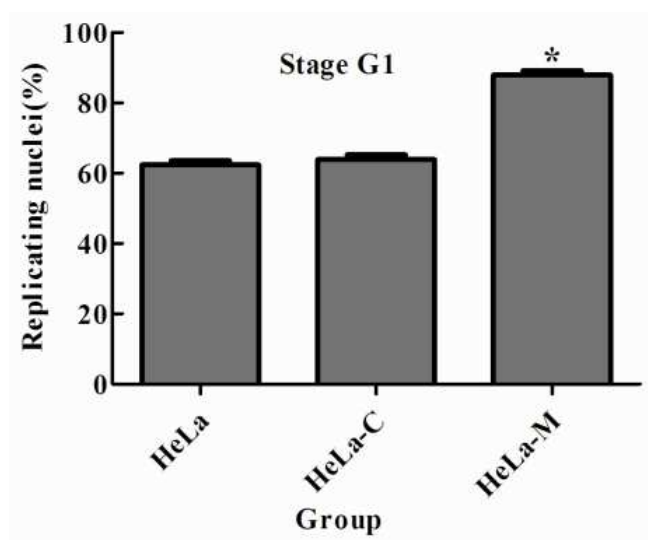

A

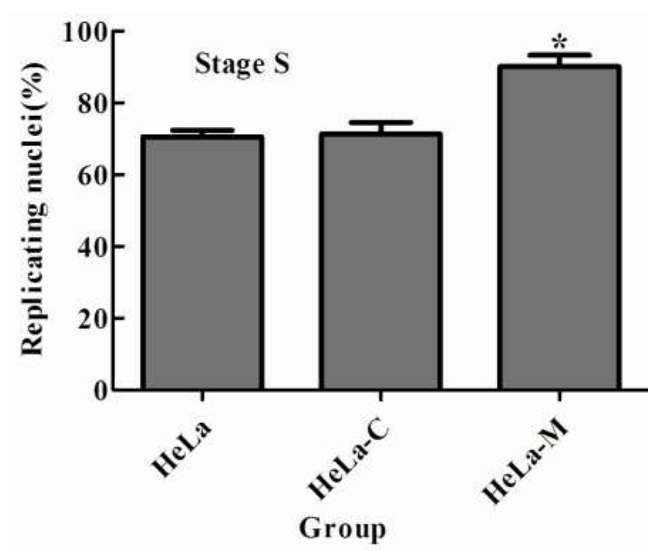

B

Figure 2. DNA replication in vitro by using mammalian cell-free DNA replication system.

The percentage of replicating nuclei in HeLa-M cells was much higher than that in HeLa cells or HeLa-C cells in Stage G1 or Stage $\mathrm{S}$ ( $\mathrm{n}=3,{ }^{*} P<0.05$ vs the other two groups).

A) The percentage of replication nuclei in stage G1. B) The percentage of replication nuclei in stage $S$.

HeLa: untransfected HeLa cells; HeLa-C: transfected pCMV/myc/nuc HeLa cells; HeLa-M: transfected pCMV/nuc/M-CSF HeLa cells.

\subsection{Effect of Nuclear M-CSF on the Cell Proliferation of HeLa Cells in Vitro and in Vivo}

The M-CSF-transfected HeLa cells had cut down doubling time $(33.38 \pm 0.19 \mathrm{~h})$ than either $\mathrm{pCMV} /$ nuc/myc-transfected $\mathrm{HeLa}$ cells $(47.92 \pm 0.22 \mathrm{~h})$ or untransfected $\mathrm{HeLa}$ cells $(47.16 \pm 0.23 \mathrm{~h})(\mathrm{n}=3, * P<0.05$ vs the other two groups, Table 2$)$.

Table 2. Effect of $n M$-CSF on the doubling time of HeLa cells. $(\overline{\boldsymbol{x}} \pm \boldsymbol{s}, n=3)$.

\begin{tabular}{llll}
\hline Time & HeLa & HeLa-C & HeLa-M \\
\hline $24 \mathrm{~h}$ & $39000 \pm 16$ & $41000 \pm 23$ & $65000 \pm 17$ \\
$48 \mathrm{~h}$ & $55500 \pm 20$ & $58000 \pm 21$ & $107000 \pm 29$ \\
Doubling time (h) & $47.16 \pm 0.23$ & $47.92 \pm 0.22$ & $33.38 \pm 0.19^{*}$ \\
\hline
\end{tabular}

HeLa: untransfected HeLa cells; HeLa-C: pCMV/nuc/myc-transfected HeLa cells; HeLa-M: pCMV/nuc/M-CSF-transfected HeLa cells.

The proliferation ability of transfected $\mathrm{pCMV} / \mathrm{nuc} / \mathrm{M}-\mathrm{CSF}$ HeLa cells was more significantly augmented than either pCMV/nuc/myc transfected HeLa cells or the untransfected cells by MTT assay (Figure 2A). What's more, M-CSF-specific antisense oligonucleotide significantly inhibited the proliferation of the M-CSF-transfected cells in a concentration-dependent manner (Figure 2B) but had little effect on the other two groups. These results suggested M-CSF expressed in nuclei could promote the cell proliferation of HeLa cells in vitro.

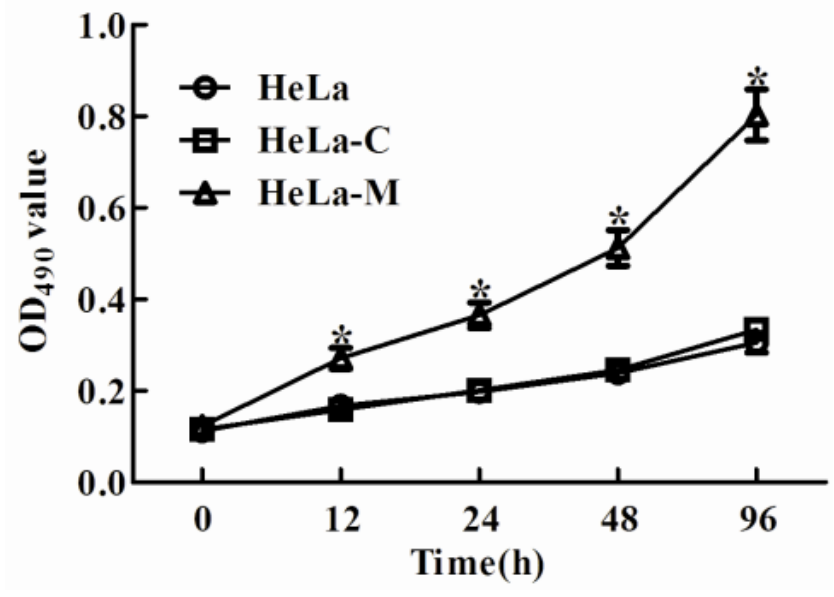

A 


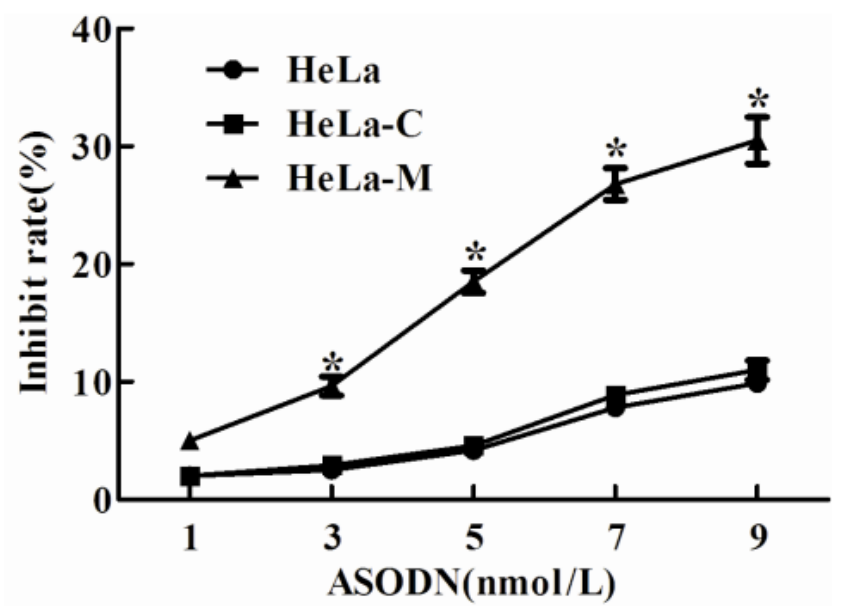

B

Figure 3. Effect of nuclear M-CSF on the cell proliferation of HeLa cells.

A) Effect of M-CSF on the viability of HeLa cells by MTT assay. The proliferation ability of transfected $\mathrm{pCMV} /$ nuc/M-CSF HeLa cells was more significantly augmented than either $\mathrm{pCMV} /$ nuc/myc transfected HeLa cells or the untransfected cells ( $\mathrm{n}=3, * P<0.05$ vs the other two groups). B) Effect of M-CSF-specific antisense oligonucleotide on the viability of pCMV/nuc/M-CSF-transfected HeLa cells. M-CSF-specific antisense oligonucleotide significantly inhibited the proliferation of the M-CSF-transfected cells in a concentration-dependent manner ( $\mathrm{n}=3, * P<0.05$ vs the other two groups), but had little effect on the other two groups.

HeLa: untransfected HeLa cells; HeLa-C: pCMV/nuc/myc-transfected HeLa cells; HeLa-M: pCMV/nuc/M-CSF-transfected HeLa cells.

When the cells were injected subcutaneously into the nude mice, the results could be seen that the xenografts formation time of the group of $\mathrm{pCMV} / \mathrm{nuc} / \mathrm{M}-\mathrm{CSF}$ transfected HeLa cells vaccinated was earlier and the tumor size and weight were increased obviously compared with the group of empty plasmid transfected or untransfected HeLa cells. The difference was statistically significant $(P<0.05)$. The time to tumor of the group of $\mathrm{pCMV} /$ nuc/M-CSF transfected HeLa cells vaccinated was earlier and the tumor weight was increased obviously than the group of empty plasmid transfected or untransfected HeLa cells, the difference was statistically significant $(P<$ $0.05)$. It indicated that nuclear M-CSF transfected in HeLa cells could promote the growth and weight of xenograft tumors in vivo (Table 3 and Figure 4). All above showed nuclear M-CSF could accelerate the cell proliferation of HeLa cells both in vitro and in vivo.

Table 3. Effect of M-CSF on the time to tumor and tumor weight of HeLa cell xenografts in nude mice.

\begin{tabular}{llll}
\hline Group & animals & $\begin{array}{l}\text { Average Time } \\
\text { to Tumor (h) }\end{array}$ & Average Tumor Weight (mg) \\
\hline HeLa & 8 & $52.25 \pm 7.83$ & $3112.21 \pm 135.37$ \\
HeLa-C & 8 & $51.49 \pm 9.21$ & $3218.46 \pm 201.52$ \\
HeLa-M & 8 & $43.26 \pm 6.34 *$ & $5293.38 \pm 319.36^{*}$ \\
\hline
\end{tabular}

*: $P<0.05$, vs the value of the groups including HeLa and HeLa-C cells. $(\overline{\boldsymbol{x}} \pm \boldsymbol{s})$.

HeLa: untransfected HeLa cells; HeLa-C: pCMV/nuc/myc-transfected HeLa cells; HeLa-M: pCMV/nuc/M-CSF-transfected HeLa cells.

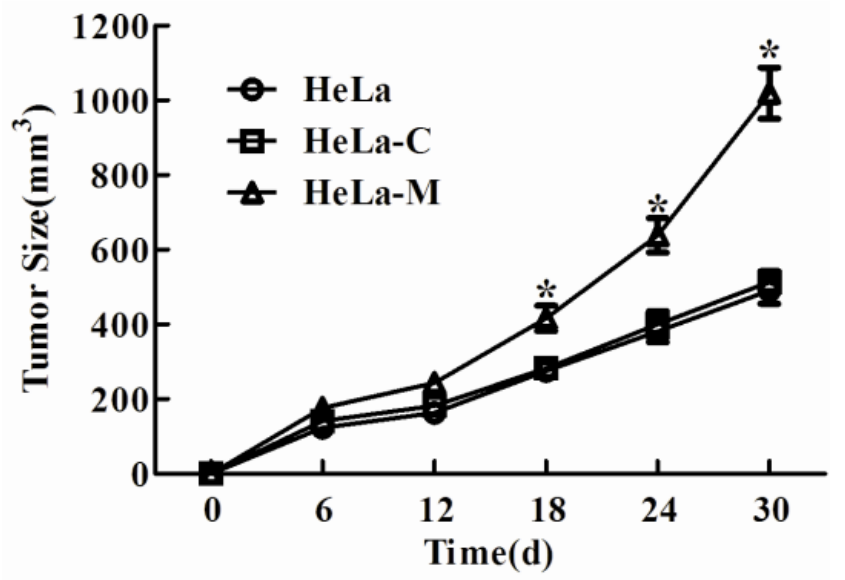

*: $P<0.05$, vs the value of the groups including HeLa and HeLa-C cells.

Figure 4. Effect of M-CSF on the tumor size of HeLa cell xenografts in nude mice.

The tumor size of the group of $\mathrm{pCMV} /$ nuc/M-CSF transfected HeLa cells vaccinated was increased obviously than the group of empty plasmid transfected or untransfected HeLa cells, the difference was statistically significant $(P<0.05)$. HeLa: untransfected HeLa cells; HeLa-C: pCMV/nuc/myc-transfected HeLa cells; HeLa-M: pCMV/nuc/M-CSF-transfected HeLa cells.

\section{Discussion}

M-CSF is an important cytokine involved in cellular signalling [21, 22]. Commonly, M-CSF secreted by cells, binds to its transmembrane receptor which leads to the transduction of the signal onto the target cell; then the M-CSF-receptor complex is internalized after accomplishing its function and degraded; therefore, M-CSF is usually not found in the cells. However, recent studies have shown that M-CSF can be expressed at a high level in the cytoplasm and nuclei of some kinds of malignant tumors [23-25]. The process is associated with enhanced tumor cells' movement and invasion, resulting in an unfavorable prognosis, which suggests that intracellular expression of M-CSF may have some specific action.

Our preliminary experiments indicated that M-CSF was expressed in HeLa cells at a low level in the cytoplasm but not seen in the nuclei, which was consistent with the literature [12]. To explore the regulation of nuclear M-CSF (nM-CSF) on the proliferation of HeLa cells, $\mathrm{pCMV} / \mathrm{nuc} / \mathrm{M}-\mathrm{CSF}$ vectors were constructed and transfected into HeLa nuclei. The results from western blot and indirect immunofluorescence showed that M-CSF protein was expressed at a higher level and localized to the nuclei in M-CSF-transfected HeLa cells compared with either $\mathrm{pCMV} / \mathrm{myc} / \mathrm{nuc}$ transfected $\mathrm{HeLa}$ cells or the untransfected HeLa cells [18]. What is the effect and mechanism of nM-CSF? Then, we analyzed the effect of nM-CSF on the proliferation of HeLa cells. M-CSF-transfected HeLa cells had cut down doubling time and more significantly augmented reproductive activity 
than the controlled HeLa cells (Table. 2). What's more, M-CSF-specific antisense oligonucleotide significantly inhibited the proliferation of the M-CSF-transfected cells in a concentration-dependent manner, but had little effect on the other two groups (Figure 3). Above all showed nM-CSF could accelerate the cell proliferation of HeLa cells in vitro. When the cells were injected subcutaneously into the nude mice, the results showed that the xenografts formation time of the group of $\mathrm{pCMV} / \mathrm{nuc} / \mathrm{M}-\mathrm{CSF}$ transfected HeLa cells vaccinated was earlier and the tumor size and weight were increased obviously than the group of empty plasmid transfected or untransfected HeLa cells (Table. 3 and Figure 4). It indicated that nuclear M-CSF transfected in HeLa cells could promote the growth and weight of xenograft tumors in vivo. So, nM-CSF could promote the cell proliferation ability of HeLa cells both in vitro and in vivo.

Cell proliferation is a complex and orderly process affected by many kinds of factors rigorously regulated. DNA replication is one of many important events in cell activities such as cell proliferation. In the process of cell division, only through the replication can genetic information contained in the parental cells be transmitted to offspring cells. After pCMV/nuc/M-CSF vectors were transfected into $\mathrm{HeLa}$ nuclei, DNA replication was assessed in vivo by BrdU and in vitro by using mammalian cell-free DNA replication system. The results showed that there was a higher percentage of replicating nuclei in the transfected pCMV/nuc/M-CSF HeLa cells both in phase G1 and $\mathrm{S}$ (Table. 1 and Figure 1 and 2).

Combining with the previous results in our study that M-CSF isolated from human leukemic cell J6-1 and HL-60 nuclei could combine with DNA, one of the non-receptor target molecules of M-CSF named MCM7 using yeast two-hybrid system from HL-60 cellular cDNA library. MCM7 is a member of MCMs. When DNA replication is initiated, MCMs are released from the chromatin. During the S-phase and until mitosis, MCMs are found unbound to replication precursors, which ensures that DNA replication happens only once during each cell cycle [26]. It has DNA helicase activity as a $(\mathrm{MCM} 4 / 6 / 7)_{2}$ dimer, and interacts with $\mathrm{Cdc} 45$ to recruit DNA polymerase $\alpha$ to the replication origin. As a result, the replication is successfully initiated [27]. Next, we would figure out the interaction between M-CSF and MCM7. Transfected M-CSF might accelerate the cell proliferation via the interaction with MCM7.

\section{Conclusion}

Therefore, the conclusion was that nM-CSF could accelerate the proliferation of HeLa cells especially in DNA replication.

\section{Acknowledgements}

This work was supported by a grant from the National Natural Science Foundation of China (30270684, 81541163), the Natural Science Youth Foundation of Hunan Province
(09JJ3060, 2015JJ3101), the Open Fund Based on Innovation Platform of Hunan Provincial Education Department (15K111), Training project of Hunan Province Cooperative innovation Center for Molecular Target New Drug Study (2014-405) and the construct program of the key discipline in Hunan province.

\section{References}

[1] Kasamatsu E, Cubilla AL, Alemany L, et al. Type-specific human papillomavirus distribution in invasive cervical carcinomas in Paraguay. A study of 432 cases. J Med Virol, 2012, 84: 1628 1635.

[2] Pandey S, Chandravati. Autophagy in cervical cancer: an emerging therapeutic target. Asian Pac J Cancer Prev, 2012, 13: 4867 4871.

[3] Ławicki S, Będkowska GE, Gacuta-Szumarska E, et al. Pretreatment plasma levels and diagnostic utility of hematopoietic cytokines in cervical cancer or cervical intraepithelial neoplasia patients. Folia Histochem Cytobiol, 2012, 50: 213 219.

[4] Beirão BC, Raposo T, Pang LY, et al. Canine mammary cancer cells direct macrophages toward an intermediate activation state between M1/M2. BMC Vet Res, 2015, 11: 151.

[5] Bellora F, Castriconi R, Doni A, et al. M-CSF induces the expression of a membrane-bound form of IL-18 in a subset of human monocytes differentiating in vitro toward macrophages. Eur J Immunol, 2012, 42: 1618 1626.

[6] Liu W, Xu GZ, Jiang CH, et al. Expression of macrophage colony-stimulating factor (M-CSF) and its receptor in streptozotocin-induced diabetic rats. Curr Eye Res, 2009, 34: $123 \sim 133$.

[7] Itoh K, Udagawa $\mathrm{N}$, Matsuzaki $\mathrm{K}$, et al. Importance of membrane- or matrix-associated forms of M-CSF and RANKL/ODF in osteoclastogenesis supported by SaOS-4/3 cells expressing recombinant $\mathrm{PTH} / \mathrm{PTHrP}$ receptors. J Bone Miner Res, 2000, 15: 1766 1775.

[8] Booker BE, Clark RS, Pellom ST, et al. Interleukin-34 induces monocytic-like differentiation in leukemia cell lines. Int $\mathrm{J}$ Biochem Mol Biol, 2015; 6 (1): 1 16.

[9] Ławicki S, Mroczko B, Omyła J, et al. Macrophage colony-stimulating factor (M-CSF) as a candidate for the tumor marker of breast cancer. Pol Arch Med Wewn 2003, 109: 597 602.

[10] Lawicki S, Bedkowska E, Gacuta-Szumarska E, et al. The plasma levels and diagnostic utility of stem cell factor (SCF) and macrophage-colony stimulating factor (M-CSF) in cervical cancer patients. Pol Merkur Lekarski, 2008, 25: 38 42.

[11] Espinosa I, Catasus L, D' Angelo E, et al. Stromal signatures in endometrioid endometrial carcinomas. Mod Pathol, 2014, 27 (4): 631 639.

[12] Kirma N, Hammes LS, Liu YG, et al. Elevated expression of the oncogene c-fms and its ligand, the macrophage colony-stimulating factor- 1 , in cervical cancer and the role of transforming growth factor-beta1 in inducing c-fms expression. Cancer Res, 2007, 67: 1918 1926. 
[13] Powell JA, Thomas D, Barry EF, et al. Expression profiling of a hemopoietic cell survival transcriptome implicates osteopontin as a functional prognostic factor in AML. Blood, 2009, 114 (23): 4859 4870.

[14] Bourguignon LY, Singleton PA, Zhu H, et al. Hyaluronan-mediated CD44 interaction with RhoGEF and Rho kinase promotes Grb2-associated binder-1 phosphorylation and phosphatidylinositol 3-kinase signaling leading to cytokine (macrophage-colony stimulating factor) production and breast tumor progression. J Biol Chem, 2003, 278: 29420 29434.

[15] Newa M, Bhandari KH, Tang L, et al. Antibody-mediated "universal" osteoclast targeting platform using calcitonin as a model drug. Pharm Res, 2011, 28: 1131 1143.

[16] Bedkowska GE, Ławicki S, Szmitkowski M. Molecular markers of carcinogenesis in the diagnostics of cervical cancer. Postepy Hig Med Dosw (Online), 2009, 63: 99 105.

[17] Mancino AT, Klimberg VS, Yamamoto M, et al. Breast cancer increases osteoclastogenesis by secreting M-CSF and upregulating RANKL in stromal cells. J Surg Res 2001, 100: $18-24$.

[18] Tu J, Teng SJ, Zhang XH, et al. Construction and identification of M-CSF stably expressed in the cellular nuclear. Chinese Journal of Pathophysiology, 2009, 25: 622 624.

[19] Cao ZY, Zhang B, Rao Q, et al. Effects of nuclear-presenting -macrophage colony-stimulating factor on the process of malignancy. Int J Hematol, 2003, 78: 87 89.

[20] Darzynkiewicz Z, Halicka HD, Zhao H, et al. Cell synchronization by inhibitors of DNA replication induces replication stress and DNA damage response: analysis by flow cytometry. Methods Mol Biol, 2011, 761: 85 96.

[21] Smith AM, Gibbons HM, Oldfield RL, et al. M-CSF increases proliferation and phagocytosis while modulating receptor and transcription factor expression in adult human microglia. $\mathrm{J}$ Neuroinflammation, 2013, 10: 85.

[22] Curry JM, Eubank TD, Roberts RD, et al. M-CSF signals through the MAPK/ERK pathway via $\mathrm{Sp} 1$ to induce VEGF production and induces angiogenesis in vivo. PLoS One, 2008, 3: e3405.

[23] Johansson AS, Lidén J, Okret S, et al. Effects of ethanol on cytokine generation and NFkappaB activity in human lung epithelial cell. Biochem Pharmacol, 2005, 70: 545 551.

[24] Lu SY, Wan HC, Li M, et al. Subcellular localization of Mitf in monocytic cells. Histochem Cell Biol, 2010, 133: 651 658.

[25] Li X, Vradii D, Gutierrez S, et al. Subnuclear targeting of Runx1 is required for synergistic activation of the myeloid specific M-CSF receptor promoter by PU. 1. J Cell Biochem, 2005, 96: 795 809.

[26] Tuteja N, Tran NQ, Dang HQ, et al. Plant MCM proteins: role in DNA replication and beyond. Plant Mol Biol, 2011, 77: 537 545.

[27] Bauerschmidt C, Pollok S, Kremmer E, et al. Interactions of human Cdc45 with the Mcm2-7 complex, the GINS complex, and DNA polymerases delta and epsilon during S phase. Genes Cells, 2007, 12: 745 758. 